\title{
Evidence for Ultra-diffuse Galaxy "Formation" through Galaxy Interactions
}

\author{
P. Bennet ${ }^{1}(1)$, D. J. Sand ${ }^{2}$ (1), D. Zaritsky ${ }^{2}$ (1) D. Crnojevic ${ }^{1,3}$, K. Spekkens ${ }^{4,5}$ (1), and A. Karunakaran ${ }^{5}$ \\ ${ }^{1}$ Physics \& Astronomy Department, Texas Tech University, Box 41051, Lubbock, TX 79409-1051, USA; paul.bennet@ttu.edu \\ ${ }^{2}$ Steward Observatory, University of Arizona, 933 North Cherry Avenue, Room N204, Tucson, AZ 85721-0065, USA \\ ${ }^{3}$ University of Tampa, 401 West Kennedy Boulevard, Tampa, FL 33606, USA \\ ${ }^{4}$ Department of Physics and Space Science, Royal Military College of Canada P.O. Box 17000, Station Forces Kingston, ON K7K 7B4, Canada \\ ${ }^{5}$ Department of Physics, Engineering Physics and Astronomy, Queen's University, Kingston, ON K7L 3N6, Canada \\ Received 2018 July 19; revised 2018 August 22; accepted 2018 September 3; published 2018 October 9
}

\begin{abstract}
We report the discovery of two ultra-diffuse galaxies (UDGs) that show clear evidence of an association with tidal material and an interaction with a larger galaxy halo, found during a search of the Wide portion of the CanadaFrance-Hawaii Telescope Legacy Survey. The two new UDGs, NGC 2708-Dw1 and NGC 5631-Dw1, are faint $\left(M_{g}=-13.7\right.$ and $\left.-11.8 \mathrm{mag}\right)$, extended $\left(r_{h}=2.60\right.$ and $\left.2.15 \mathrm{kpc}\right)$, and have low central surface brightness $\left(\mu(g, 0)=24.9\right.$ and $\left.27.3 \mathrm{mag} \operatorname{arcsec}^{-2}\right)$, while the stellar stream associated with each has a surface brightness $\mu(g) \gtrsim 28.2 \mathrm{mag} \mathrm{arcsec}^{-2}$. These observations provide evidence that the origin of some UDGs may connect to galaxy interactions, either by transforming normal dwarf galaxies by expanding them, or because UDGs can collapse out of tidal material (i.e., they are tidal dwarf galaxies). Further work is needed to understand the fraction of the UDG population "formed" through galaxy interactions, and wide field searches for diffuse dwarf galaxies will provide further clues to the origin of these enigmatic stellar systems.
\end{abstract}

Key words: galaxies: dwarf - galaxies: evolution - galaxies: formation

\section{Introduction}

The last several years have seen a resurgence of interest in the low surface brightness universe, and in particular the population of so-called ultra-diffuse galaxies (UDGs; van Dokkum et al. 2015), a term that refers to the largest, lowest surface brightness objects, with half-light radii $>1.5 \mathrm{kpc}$ and central surface brightnesses $>24$ mag $\operatorname{arcsec}^{-2}$. Although UDGs have been discussed in the literature for some time (e.g., Sandage \& Binggeli 1984; Caldwell \& Bothun 1987; Impey et al. 1988; Dalcanton et al. 1997; Conselice et al. 2003, among others), recent work has found hundreds of examples in cluster environments (Koda et al. 2015; Mihos et al. 2015; Muñoz et al. 2015; van Dokkum et al. 2015; van der Burg et al. 2016; Yagi et al. 2016), along with lower density groups (Crnojević et al. 2016; Merritt et al. 2016; Toloba et al. 2016; Román \& Trujillo 2017; Cohen et al. 2018; Spekkens \& Karunakaran 2018) and field examples (Martínez-Delgado et al. 2016; Bellazzini et al. 2017; Kadowaki et al. 2017; Leisman et al. 2017).

There is considerable debate as to the origin of UDGs, and it is likely that they are a "mixed bag" of populations with multiple origins (e.g., Zaritsky 2017; Lim et al. 2018). For instance, some UDGs may be "failed galaxies" with Milky Way-like total masses, but with dwarf galaxy stellar masses (e.g., van Dokkum et al. 2015, 2016; Trujillo et al. 2017), while others appear to simply be the low surface brightness extension of the standard dwarf galaxy population (Beasley \& Trujillo 2016; Amorisco et al. 2018; Sifón et al. 2018). Most UDGs with metallicity measurements point to a dwarf galaxy origin consistent with their metal-poor stellar populations (e.g., Kadowaki et al. 2017; Ferré-Mateu et al. 2018; Pandya et al. 2018). Different formation scenarios posit that UDGs have been subject to extreme feedback, which inhibited early star formation (Di Cintio et al. 2017; Chan et al. 2018), or that they are the "high-spin" tail of the dwarf galaxy population (Amorisco \& Loeb 2016). A more prosaic explanation would be that UDGs are the product of tidal and/or ram pressure stripping (e.g., Conselice 2018), which can remove stars and expand the galaxy's size (e.g., Errani et al. 2015); semi-analytic calculations show that this scenario is viable for cluster UDGs (Carleton et al. 2018). Similarly, although this has rarely been discussed in the literature (although see Trujillo et al. 2018, and their discussion of NGC 1052-DF2; van Dokkum et al. 2018b), some UDGs could plausibly be large, low surface brightness tidal dwarf galaxies (TDGs). Born during gas-rich galaxy collisions, TDGs should generally be lacking in dark matter and be metal-rich in comparison to normal dwarfs of the same luminosity (e.g., Hunsberger et al. 1996; Duc 2012, among many others). This could be a way to produce a dark matterfree UDG, such as is claimed for NGC 1052-DF1 (van Dokkum et al. 2018b); however, in that case interpretation is still under extensive discussion and the presence of a globular cluster (GC) population (van Dokkum et al. 2018a) is a significant problem for a TDG interpretation. Observationally, some TDGs can survive for $\sim 4 \mathrm{Gyr}$, and have size and surface brightness properties similar to the recently identified UDG class of galaxies (Duc et al. 2014).

There is some observational evidence for a UDG "galaxy interaction" formation scenario in the radial alignment of Coma UDGs (Yagi et al. 2016), the kinematics of the GCs in at least one Virgo UDG (Toloba et al. 2018), and in the very elongated UDG associated with NGC 253 (Scl-MM-Dw2; Toloba et al. 2016). Other UDG-like systems also have suggestive features pointing to a recent galaxy interaction (e.g., Martínez-Delgado et al. 2012; Merritt et al. 2016; Greco et al. 2018a), or even spatial/kinematic substructure that could result from such interactions (e.g., And XIX; Collins et al. 2013). To our knowledge, the only direct observational evidence that UDGlike objects can form from galaxy interactions comes from (a) the disrupting dwarf, CenA-MM-Dw3, which has a $r_{\text {half }}=2.5 \mathrm{kpc}$ and $\mu_{0}=26.0 \mathrm{mag} \mathrm{arcsec}^{-2}$, with clear tidal streams extending over $\sim 60 \mathrm{kpc}$ in the outskirts of the nearby 
elliptical Centaurus A (Crnojević et al. 2016) and (b) VLSB-A, a nucleated Virgo UDG that has clear tidal features, and is possibly associated with M86 (Mihos et al. 2015).

Here we present two additional UDGs discovered during a semi-automated, ongoing search for diffuse dwarf galaxies in the Wide portion of the Canada-France-Hawaii Telescope Legacy Survey (CFHTLS)—see Bennet et al. (2017) for initial results around M101, and a description of our algorithm. Both UDGs show associated stellar streams connected to a parent galaxy halo, suggesting that they are being shaped by ongoing galaxy interactions. This further, direct observational evidence that UDGs can be the product of interactions suggests that this is a viable formation channel for this enigmatic galaxy population.

\section{The Data and UDG Detection}

We are searching for diffuse galaxies in the Wide portion of the CFHTLS, concentrating on fields W1, W2, and W3, using an updated version of the semi-automated detection algorithm presented in Bennet et al. (2017). The total area being searched is $\sim 150 \mathrm{deg}^{2}$. The CFHTLS data was taken with the $\sim 1 \times$ $1 \mathrm{deg}^{2}$ MegaPrime imager (Boulade et al. 2003), with typical exposure times for each field of $\sim 2750$ and $2500 \mathrm{~s}$ in the $g$ and $r$ bands, respectively. The fields were downloaded directly from the Canadian Astronomy Data Centre, as were the pointspread functions (PSFs) for those image stacks, which were used for measuring dwarf structural parameters and simulating injected dwarfs. The construction and calibration of these stacks used the MegaPipe data pipeline (Gwyn 2008), and is described in detail by Gwyn (2012).

Here we briefly outline our diffuse dwarf detection algorithm, which has been updated slightly from that presented in Bennet et al. (2017); the algorithm borrows elements from previous work (e.g., Dalcanton et al. 1997; Davies et al. 2016; van der Burg et al. 2016). All diffuse dwarf detection is done on the $g$-band stacked images from the CFHTLS. First, bright stars and galaxies are directly masked by matching source positions with the Guide Star Catalog 2.3.2 (Lasker et al. 2008), and then fainter objects are identified and masked by a call to SExtractor (Bertin \& Arnouts 1996). This step leaves only very faint objects ( $<3 \sigma$ above the background), extended galaxy halos, and low surface brightness features remaining in the image. After masking, each image is binned by $150 \times 150$ pixels $(28 \times 28$ arcsec $)$, a spatial scale chosen to maximize the detection of large, diffuse objects while also remaining sensitive to smaller features. Another round of SExtractor is run to identify objects on the binned images, and all candidates are forwarded for visual inspection via a web interface, where our final diffuse dwarf candidates are selected.

We implant simulated dwarf galaxies directly into our images before performing the search in order to better characterize our detection efficiency. Simulated dwarfs are injected in batches of 10, randomly placed throughout each image. Each simulated dwarf has a Sérsic profile (Sersic 1968) with index $n=0.5-2.0$ and ellipticity $0.0-0.7$, randomly chosen, which is representative of past UDG measurements. Each dwarf is given a $g$-band magnitude between $g=$ $16-23 \mathrm{mag}$, with half-light radii in the range $\approx 2-370$ arcsec. This range of parameters spans that of normal galaxies to the ultra-diffuse (although this statement is distance dependent), and allows a true quantification of our detection efficiency. Roughly speaking, given our current binning scale for the
CFHTLS data, we are $\sim 90 \%$ complete down to a central surface brightness of $\approx 28.0 \mathrm{mag} \operatorname{arcsec}^{-2}$ for objects that are brighter than $g \approx 22 \mathrm{mag}-$ we will present our complete detection efficiency results in an upcoming work (P. Bennet et al. 2018, in preparation).

While still in progress, our search of the CFHTLS Wide fields have uncovered hundreds of diffuse dwarf candidates, dozens of which are likely UDGs. We will present their demographics in an upcoming work, and compare our results with other wide field searches (e.g., Greco et al. 2018b). Here we present two remarkable UDGs which clearly show signs of interaction and stripping, either of the UDG or the parent halo, likely pointing directly to their formation mechanism.

\section{Results}

During our ongoing search of the CFHTLS, two clear UDG examples exhibited stellar streams connecting them to a parent galaxy halo. We show NGC 5631-Dw1 and NGC 2708-Dw1 in Figures 1 and 2, respectively, including masked and binned versions to highlight the stream associated with each dwarf. We assume that each object is at the distance of its parent galaxy$D=40.6$ and $D=28.4 \mathrm{Mpc}$ for NGC 2708-Dw1 and NGC 5631-Dw1, respectively, based on a Tully-Fisher distance for NGC 2708 and surface brightness fluctuations for NGC 5631 (Tully et al. 2013; Courtois et al. 2011, respectively). These distances will have their own associated uncertainty that will effect the inferred physical size and luminosity of each dwarf, although the surface brightness will remain unchanged.

\subsection{Structure and Luminosity}

The observational parameters for each UDG were derived using GALFIT (Peng et al. 2002), while the uncertainties were determined by implanting 100 simulated dwarfs with the bestfit properties into our images and re-measuring each with GALFIT; the scatter in these measurements is our quoted uncertainty (see Merritt et al. 2014; Bennet et al. 2017). Both objects were fit with a standard Sérsic profile (Sersic 1968). We allowed all parameters to vary without restriction for NGC 2708-Dw1, but fixed the Sérsic index to $n=1$ for NGC 5631Dw1 to facilitate the fit, given its extremely low surface brightness. As these objects were very low surface brightness, spatial binning was required. It is also difficult to disentangle the dwarf and its associated stream in the GALFIT process, and there may be an additional systematic uncertainty related to this, although on visual inspection the fits are excellent. We show our fits and residuals in Figures 1 and 2.

We put these newly found UDGs in context with those in the literature in Figure 3, where we compare them with the UDGs found in Coma (van Dokkum et al. 2015), and the H I-rich UDG sample of Leisman et al. (2017). NGC 2708-Dw1 has properties that are typical of the general Coma UDG population, with $r_{h}=2.60 \pm 0.57 \mathrm{kpc}, M_{g}=-13.7 \pm 0.3 \mathrm{mag}$ and a central surface brightness of $\mu(g, 0)=24.9 \pm 0.6 \mathrm{mag} \mathrm{arcsec}^{-2}$. NGC $5631-\mathrm{Dw} 1$, with a $r_{h}=2.15 \pm 0.50 \mathrm{kpc}, \quad M_{g}=-11.8 \pm$ $0.4 \mathrm{mag}$ and a central surface brightness of $\mu(\mathrm{g}, 0)=27.3 \pm$ $0.6 \mathrm{mag} \operatorname{arcsec}^{-2}$; however, is relatively unique and stands out for its very faint central surface brightness. Many objects of similarly low surface brightness are found in our general CFHTLS search, and we expect to fill in this surface brightness range in future work. We also plot the two UDGs in the Local Universe that also show signs of interaction-Scl-MM-Dw2 


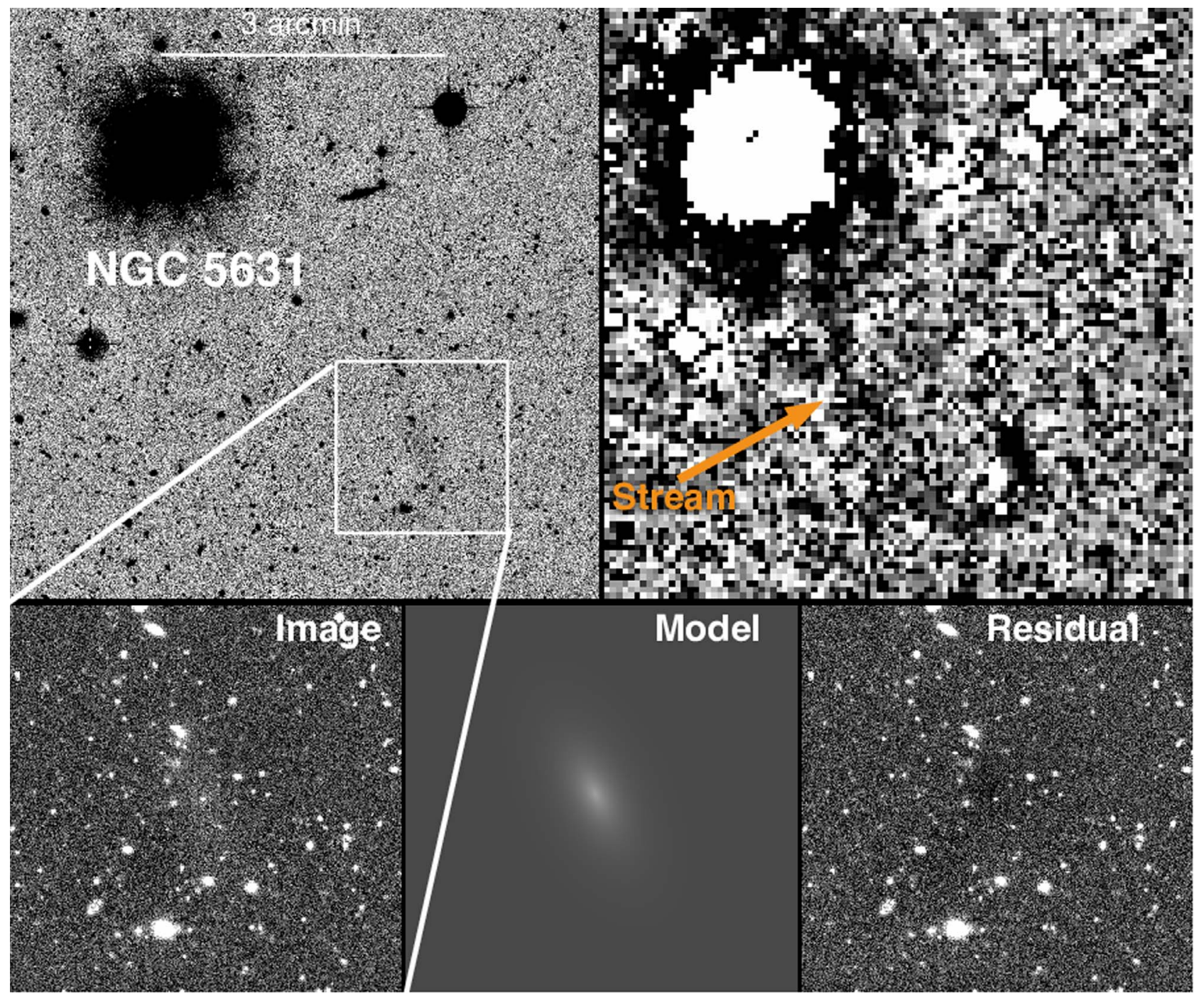

Figure 1. $g$-band CFHTLS data of NGC 5631-Dw1; North is up and East is to the left in all panels. The upper-left image shows the CFHTLS image at full resolution (where the stream is not visible, but NGC 5631-Dw1 is just apparent), while the upper-right image has been binned and masked to enhance low surface brightness features. The lower-left panel shows a zoomed in $g$-band image of NGC 5631-Dw1, the lower-middle panel shows the GALFIT model, and the lower-right panel shows the residuals. A clear but very faint stellar stream trails behind the UDG as a dark feature in the binned and masked image.

Toloba et al. (2016) and CenA-MM-Dw3 (Crnojević et al. 2016).

We checked Galaxy Evolution Explorer (GALEX; Martin \& GALEX Team 2005) imaging at the position of each dwarf, finding no near-ultraviolet/far-ultraviolet (NUV/FUV) emission for either object. From these $\sim 1500 \mathrm{~s}$ exposures, we derive NUV $>20.9$ and $>20.7$ mag for NGC 2708-Dw1 and NGC 5631-Dw1, respectively, and derive a limit on the star formation rate of $\lesssim 3.1 \times 10^{-3}$ and $\lesssim 3.3 \times 10^{-3} M_{\odot} \mathrm{yr}^{-1}$ (Iglesias-Páramo et al. 2006) for each object in turn. The $g-r$ color of the two UDGs are quite uncertain (see Table 1), but given the lack of GALEX detections for each object, they are likely passively evolving at the present epoch (see also the brief $\mathrm{H}$ I discussion below).

We estimate the average surface brightness of the streams associated with NGC 2708-Dw1 and NGC 5631-Dw1 by taking a polygon over the stream area, and aggressively masking intervening, bright sources. The NGC 2708-Dw1 stream is at $\mu(g) \sim 28.2 \mathrm{mag} \operatorname{arcsec}^{-2}$, while that of NGC
5631-Dw1 is $\mu(g) \sim 28.4 \mathrm{mag} \operatorname{arcsec}^{-2}$. These streams are extremely faint, and may be why similar structures are not more routinely seen around UDGs.

\subsection{Environment}

Both NGC 2708-Dw1 and NGC 5631-Dw1 are found in a group environment, which is conducive to galaxy encounters (e.g., Barnes 1985) and may point to the role that groups play in building up the UDG population across halo masses.

NGC 5631 is an elliptical galaxy, and member of a loose group (Geller \& Huchra 1983; Pisano et al. 2004) that is also composed of NGC 5667 and NGC 5678, and possibly several other fainter galaxies. The H I study of Serra et al. (2012) shows an H I extension to the southwest of NGC 5631 in the general direction of NGC 5631-Dw1; however, this stops short of the position of NGC 5631-Dw1 and is not aligned with the stream. This lack of H I (with a limit of $M_{\mathrm{H} \mathrm{I}} \lesssim 5 \times 10^{7} M_{\odot}$ ) within the UDG corroborates the GALEX observations, which indicate that it is not actively forming stars. 


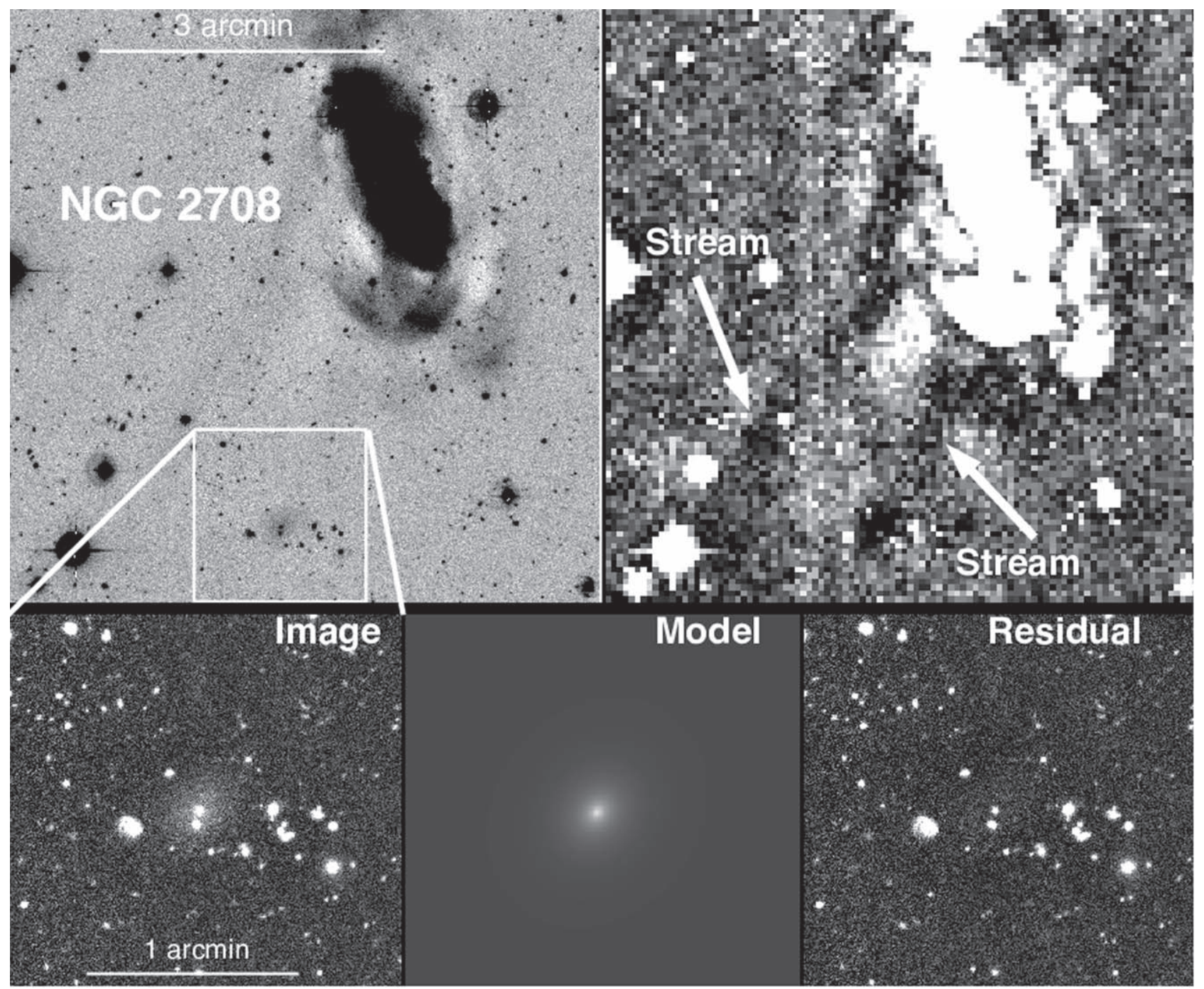

Figure 2. $g$-band CFHTLS data of NGC 2708-Dw1; North is up and East is to the left in all panels. The upper-left image shows the CFHTLS image at full resolution, while the upper-right image has been binned and masked to enhance low surface brightness features. Two streams are the dark features apparent in the binned +masked image, one connecting NGC 2708-Dw1 to the main body of NGC 2708, and another longer stream just to the North of it. Elements of both streams are also visible in the full-resolution image. The lower-left panel shows a zoomed in $g$-band image of NGC 2708-Dw1, the lower-middle panel shows the GALFIT model, and the lower-right panel shows the residuals.

The spiral galaxy NGC 2708 is a member of the "NGC 2698 group" as identified by Makarov \& Karachentsev (2011), which has a group velocity dispersion of $\sigma=94 \mathrm{~km} \mathrm{~s}^{-1}$ and eight identified members. NGC 2708 itself has undergone several interactions beyond those associated with NGC 2708Dw1. There is a separate, long tidal stream $(\sim 50 \mathrm{kpc})$ directly to the north of NGC 2708-Dw1 that is visible in Figure 2, which terminates at the same location as a bright foreground star in the southeast portion of the figure. There is yet another stream which emanates to the north of NGC 2708 (not pictured in Figure 2), approximately $26 \mathrm{kpc}$ long, which also terminates in a fluffy, dwarf-like structure (its morphology is somewhat reminiscent of the "dog leg stream" in NGC 1097; Galianni et al. 2010). Portions of this northern stream have been identified previously, and Very Large Array observations reveal it to be H I-rich (Pisano et al. 2002) - these same H I observations do not show any H I associated with NGC 2708Dw1, with a limit of $M_{\mathrm{H} \mathrm{I}} \lesssim 10^{7} M_{\odot}$ (Pisano et al. 2002), bolstering our argument that this galaxy is no longer forming stars.

\section{Summary and Conclusions}

We have presented the discovery of two new UDGs with clear evidence for associated stellar streams due to encounters with nearby massive galaxies. The main body of each dwarf is consistent with the general UDG population (although NGC 5631-Dw1 is fainter and lower surface brightness than the bulk of the population), while the stellar streams have estimated surface brightnesses of $\mu(\mathrm{g}) \gtrsim 28.2 \mathrm{mag} \mathrm{arcsec}{ }^{-2}$. Both UDGs are likely dominated by old, passively evolving stellar populations and reside in a group environment, similar to other (but not all) UDG discoveries. These stripped objects, along with UDGs discovered in the nearby universe via resolved stellar surveys, point to a possible "formation mechanism" for some fraction of the UDG population.

A scenario where UDGs are produced by galaxy interactions was recently presented by Carleton et al. (2018), and has been suggested elsewhere (e.g., Conselice 2018). In their work, Carleton et al. (2018) performed semi-analytic calculations of dwarf galaxies (with both cuspy and cored dark matter halos) in a cluster environment. Dwarf galaxies with cored dark matter 

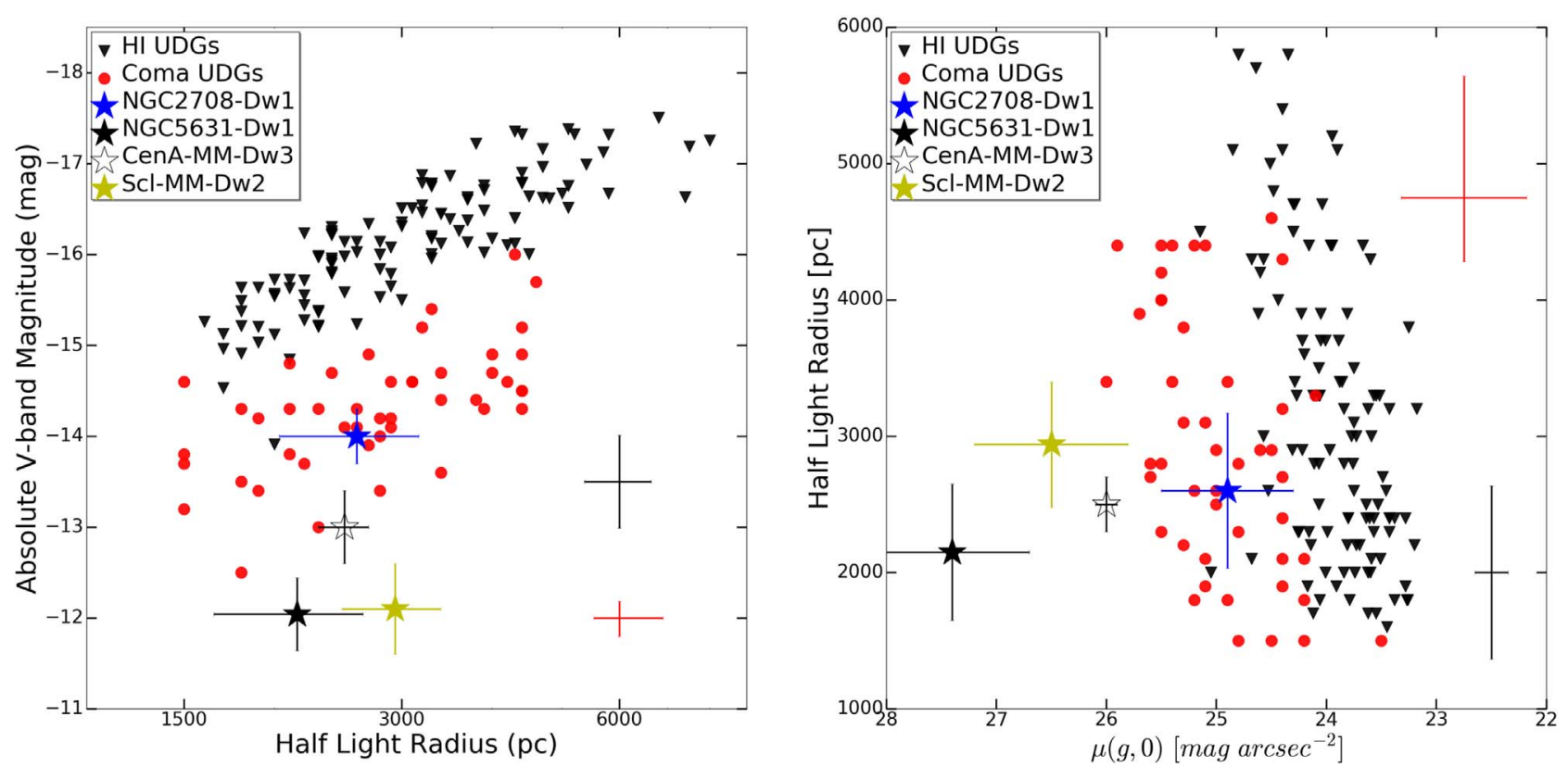

Figure 3. Left panel: the size-luminosity relation for NGC 2708-Dw1 and NGC 5631-Dw1 (stars) compared to other UDG populations-H I-rich UDGs (Leisman et al. 2017) are shown as inverted black triangles, while the Coma UDGs (van Dokkum et al. 2015) are shown as red circles. Typical errors for each population are shown on the right of each plot. Also shown are CenA-MM-Dw3 (Crnojević et al. 2016), Scl-MM-Dw2 (Toloba et al. 2016), and the two Local Volume UDGs that show clear signs of disruption. Where direct V-band observations were unavailable they were derived from $g$-band and $r$-band data via the procedure in Jester et al. (2005). Right panel: the central surface brightness as a function of half-light radius for our newly discovered UDGs, plotted with the other UDG populations.

Table 1

Stripped UDG Properties

\begin{tabular}{lcc}
\hline \hline Name & NGC 2708-Dw1 & NGC 5631-Dw1 \\
\hline R.A. (J2000) & $08: 56: 12.7$ & $14: 26: 13.6$ \\
Decl. (J2000) & $-03: 25: 14.8$ & $+56: 31: 50.2$ \\
$m_{g}$ (mag) & $19.3 \pm 0.3$ & $20.5 \pm 0.4$ \\
$M_{g}(\mathrm{mag})$ & $-13.7 \pm 0.3$ & $-11.8 \pm 0.4$ \\
Color $(g-r)$ & $0.5 \pm 0.4$ & $0.4 \pm 0.6$ \\
$r_{h}(\operatorname{arcsec})$ & $13.2 \pm 2.9$ & $15.6 \pm 3.6$ \\
$r_{h}(\mathrm{kpc})$ & $2.60 \pm 0.57$ & $2.15 \pm 0.50$ \\
Sérsic index & $1.48 \pm 0.15$ & $1.00^{\mathrm{a}}$ \\
Axis ratio & $0.83 \pm 0.05$ & $0.54 \pm 0.09$ \\
$\mu(g, 0)\left(\right.$ mag $\left.\operatorname{arcsec}^{-2}\right)$ & $24.9 \pm 0.6$ & $27.3 \pm 0.7$ \\
$D$ (Mpc) & 40.6 & 28.4 \\
Projected distance $(\mathrm{kpc})$ & 45.2 & 34.1 \\
\hline
\end{tabular}

Note.

a The Sérsic index for NGC 5631-Dw1 is fixed to $n=1$; see the text for details.

profiles were preferentially shaped by galaxy interactions, causing their stellar mass to decrease and half-light radii to increase, and the team was able to reproduce the demographics of the cluster UDG population. It should be noted that Carleton et al. (2018) did not recover the observed cluster UDG population with cuspy dwarf galaxy halos, although individual objects did take on UDG-like properties. While these calculations were specifically done for a cluster environment, they should also be applicable to group environments such as that observed in the current work.

The UDGs in this Letter could also be TDGs, the dark matter-free product of gas-rich galaxy interactions that continue as cohesive stellar units (for a recent review, see Duc 2012), and which some observations have shown can be relatively long lived ( 4 Gyr; Duc et al. 2014). While NGC 5631-Dw1 and NGC 2708-Dw1 are both associated with the ends of stellar stream material, as might be expected from a TDG scenario, neither has associated $\mathrm{HI}$ gas, which seems to be a ubiquitous TDG feature unless the system is very old (although the NGC 2708 system appears to have several ongoing encounters, at least one of which is gas rich). A deep search for neutral gas associated with these UDGs would help clarify their origins. A TDG origin for these objects could also be shown in the massmetallicity relation; TDGs should be metal-rich compared to equivalent stellar mass dwarf galaxies as they are formed from pre-enriched material from the outskirts of a disk rather than primordial gas (Hunter et al. 2000).

It is also possible that the systems discovered in this Letter are not long-lived structures, and are TDG-like enhancements in tidal streams that match the photometric criteria for UDGs. In this case, it is possible that a portion of the UDG population are chance enhancements of otherwise regular tidal features.

Additionally, future Hubble Space Telescope follow-up accounting of the GC population for these and other UDG systems may also distinguish between formation scenarios-a TDG origin would have few or no associated star clusters, normal dwarfs would have a few GCs (e.g., Zaritsky et al. 2016, with the caveat that these may be getting stripped in the interactions associated with NGC 5631-Dw1 and NGC 2708-Dw1), while more massive UDGs would have commensurately more associated GCs (Beasley \& Trujillo 2016; van Dokkum et al. 2016).

While local, resolved stellar searches for dwarfs have turned up UDGs that show signs of disturbance (Crnojević et al. 2016; Toloba et al. 2016), direct searches for classical "S"-shaped morphologies among the Coma UDGs have not revealed such tidal features (Mowla et al. 2017, although see VLSB-A in the Virgo cluster; Mihos et al. 2015), although the authors admit that they are not sensitive to all signs of tidal disturbance (see further discussions in Yagi et al. 2016; Burkert 2017; Venhola et al. 2017). In any 
case, it is not clear how long the stellar streams seen in this Letter would be visible, as stream lifetimes depend on the dwarf velocity dispersion, stellar radius, and orbital eccentricity (see the discussion in Peñarrubia et al. 2009)_further modeling of the persistence of tidal features around UDGs in a "galaxy interaction" scenario would help constrain the fraction of the population that forms in this manner.

It is not likely that galaxy interactions can explain the entirety of the UDG population, as an abundant number of field UDGs have been identified (e.g., Leisman et al. 2017) that have likely never encountered another galaxy. Note that we also cannot rule out a scenario where our UDGs formed by some other mechanism (e.g., van Dokkum et al. 2015; Amorisco \& Loeb 2016; Di Cintio et al. 2017, as discussed in Section 1), and have subsequently undergone interactions with a larger primary galaxy-by the same token, one can no longer refute the "galaxy interaction" UDG hypothesis by stating that UDGs show no sign of stripping or interaction. Future wide-field searches for diffuse dwarf galaxies will reveal their demographics across environments, and hopefully shed light on the origin of the entirety of the UDG population.

Research by D.J.S. is supported by NSF grants AST1821967, 1821987, 1813708, and 1813466. K.S. acknowledges support from the Natural Sciences and Engineering Research Council of Canada (NSERC). D.Z. gratefully acknowledges financial support through NSF AST-1713841. Research by D.C. is supported by NSF grants AST-1814208.

Based on observations obtained with MegaPrime/MegaCam, a joint project of CFHT and CEA/IRFU, at the CanadaFrance-Hawaii Telescope (CFHT) which is operated by the National Research Council (NRC) of Canada, the Institut National des Science de l'Univers of the Centre National de la Recherche Scientifique (CNRS) of France, and the University of Hawaii. This work is based in part on data products produced at Terapix available at the Canadian Astronomy Data Centre as part of the Canada-France-Hawaii Telescope Legacy Survey, a collaborative project of NRC and CNRS.

Facility: Canada-France-Hawaii Telescope (Megacam).

Software: astropy (Astropy Collaboration et al. 2013; The Astropy Collaboration et al. 2018), SExtractor (Bertin \& Arnouts 1996), GALFIT (Peng et al. 2002).

\section{ORCID iDs}

P. Bennet (i) https://orcid.org/0000-0001-8354-7279

D. J. Sand (iD https://orcid.org/0000-0003-4102-380X

D. Zaritsky (i) https://orcid.org/0000-0002-5177-727X

K. Spekkens (iD https://orcid.org/0000-0002-0956-7949

\section{References}

Amorisco, N. C., \& Loeb, A. 2016, MNRAS, 459, L51

Amorisco, N. C., Monachesi, A., Agnello, A., \& White, S. D. M. 2018, MNRAS, 475, 4235

Astropy Collaboration, Robitaille, T. P., Tollerud, E. J., et al. 2013, A\&A, 558, A33

Barnes, J. 1985, MNRAS, 215, 517

Beasley, M. A., \& Trujillo, I. 2016, ApJ, 830, 23

Bellazzini, M., Belokurov, V., Magrini, L., et al. 2017, MNRAS, 467, 3751

Bennet, P., Sand, D. J., Crnojević, D., et al. 2017, ApJ, 850, 109

Bertin, E., \& Arnouts, S. 1996, A\&AS, 117, 393

Boulade, O., Charlot, X., Abbon, P., et al. 2003, Proc. SPIE, 4841, 72

Burkert, A. 2017, ApJ, 838, 93

Caldwell, N., \& Bothun, G. D. 1987, AJ, 94, 1126
Carleton, T., Errani, R., Cooper, M., Kaplinghat, M., \& Peñarrubia, J. 2018, arXiv: 1805.06896

Chan, T. K., Kereš, D., Wetzel, A., et al. 2018, MNRAS, 478, 906

Cohen, Y., van Dokkum, P., Danieli, S., et al. 2018, arXiv:1807.06016

Collins, M. L. M., Chapman, S. C., Rich, R. M., et al. 2013, ApJ, 768, 172

Conselice, C. J. 2018, RNAAS, 2, 43

Conselice, C. J., Gallagher, J. S., III, \& Wyse, R. F. G. 2003, AJ, 125, 66

Courtois, H. M., Tully, R. B., \& Héraudeau, P. 2011, MNRAS, 415, 1935

Crnojević, D., Sand, D. J., Spekkens, K., et al. 2016, ApJ, 823, 19

Dalcanton, J. J., Spergel, D. N., Gunn, J. E., Schmidt, M., \& Schneider, D. P. 1997, AJ, 114, 635

Davies, J. I., Davies, L. J. M., \& Keenan, O. C. 2016, MNRAS, 456, 1607

Di Cintio, A., Brook, C. B., Dutton, A. A., et al. 2017, MNRAS, 466, L1

Duc, P.-A. 2012, ASSP, 28, 305

Duc, P.-A., Paudel, S., McDermid, R. M., et al. 2014, MNRAS, 440, 1458

Errani, R., Peñarrubia, J., \& Tormen, G. 2015, MNRAS, 449, L46

Ferré-Mateu, A., Alabi, A., Forbes, D. A., et al. 2018, MNRAS, 479, 4891

Galianni, P., Patat, F., Higdon, J. L., Mieske, S., \& Kroupa, P. 2010, A\&A, 521, A20

Geller, M. J., \& Huchra, J. P. 1983, ApJS, 52, 61

Greco, J. P., Greene, J. E., Price-Whelan, A. M., et al. 2018a, PASJ, 70, S19

Greco, J. P., Greene, J. E., Strauss, M. A., et al. 2018b, ApJ, 857, 104

Gwyn, S. D. J. 2008, PASP, 120, 212

Gwyn, S. D. J. 2012, AJ, 143, 38

Hunsberger, S. D., Charlton, J. C., \& Zaritsky, D. 1996, ApJ, 462, 50

Hunter, D. A., Hunsberger, S. D., \& Roye, E. W. 2000, ApJ, 542, 137

Iglesias-Páramo, J., Buat, V., Takeuchi, T. T., et al. 2006, ApJS, 164, 38

Impey, C., Bothun, G., \& Malin, D. 1988, ApJ, 330, 634

Jester, S., Schneider, D. P., Richards, G. T., et al. 2005, AJ, 130, 873

Kadowaki, J., Zaritsky, D., \& Donnerstein, R. L. 2017, ApJL, 838, L21

Koda, J., Yagi, M., Yamanoi, H., \& Komiyama, Y. 2015, ApJL, 807, L2

Lasker, B. M., Lattanzi, M. G., McLean, B. J., et al. 2008, AJ, 136, 735

Leisman, L., Haynes, M. P., Janowiecki, S., et al. 2017, ApJ, 842, 133

Lim, S., Peng, E. W., Côté, P., et al. 2018, ApJ, 862, 82

Makarov, D., \& Karachentsev, I. 2011, MNRAS, 412, 2498

Martin, C. \& GALEX Team 2005, in IAU Symp. 216, Maps of the Cosmos, ed. M. Colless, L. Staveley-Smith, \& R. A. Stathakis (San Francisco, CA: ASP), 221

Martínez-Delgado, D., Läsker, R., Sharina, M., et al. 2016, AJ, 151, 96

Martínez-Delgado, D., Romanowsky, A. J., Gabany, R. J., et al. 2012, ApJL, 748, L24

Merritt, A., van Dokkum, P., \& Abraham, R. 2014, ApJL, 787, L37

Merritt, A., van Dokkum, P., Danieli, S., et al. 2016, ApJ, 833, 168

Mihos, J. C., Durrell, P. R., Ferrarese, L., et al. 2015, ApJL, 809, L21

Mowla, L., van Dokkum, P., Merritt, A., et al. 2017, ApJ, 851, 27

Muñoz, R. P., Eigenthaler, P., Puzia, T. H., et al. 2015, ApJL, 813, L15

Pandya, V., Romanowsky, A. J., Laine, S., et al. 2018, ApJ, 858, 29

Peñarrubia, J., Navarro, J. F., McConnachie, A. W., \& Martin, N. F. 2009, ApJ, 698, 222

Peng, C. Y., Ho, L. C., Impey, C. D., \& Rix, H.-W. 2002, AJ, 124, 266

Pisano, D. J., Wakker, B. P., Wilcots, E. M., \& Fabian, D. 2004, AJ, 127, 199

Pisano, D. J., Wilcots, E. M., \& Liu, C. T. 2002, ApJS, 142, 161

Román, J., \& Trujillo, I. 2017, MNRAS, 468, 4039

Sandage, A., \& Binggeli, B. 1984, AJ, 89, 919

Serra, P., Oosterloo, T., Morganti, R., et al. 2012, MNRAS, 422, 1835

Sersic, J. L. 1968, Atlas de Galaxias Australes

Sifón, C., van der Burg, R. F. J., Hoekstra, H., Muzzin, A., \& Herbonnet, R. 2018, MNRAS, 473, 3747

Spekkens, K., \& Karunakaran, A. 2018, ApJ, 855, 28

The Astropy Collaboration, Price-Whelan, A. M., Sipőcz, B. M., et al. 2018, arXiv:1801.02634

Toloba, E., Lim, S., Peng, E., et al. 2018, ApJL, 856, L31

Toloba, E., Sand, D., Guhathakurta, P., et al. 2016, ApJL, 830, L21

Trujillo, I., Beasley, M. A., Borlaff, A., et al. 2018, arXiv:1806.10141

Trujillo, I., Roman, J., Filho, M., \& Sánchez Almeida, J. 2017, ApJ, 836, 191

Tully, R. B., Courtois, H. M., Dolphin, A. E., et al. 2013, AJ, 146, 86 van der Burg, R. F. J., Muzzin, A., \& Hoekstra, H. 2016, A\&A, 590, A20

van Dokkum, P., Abraham, R., Brodie, J., et al. 2016, ApJL, 828, L6 van Dokkum, P., Cohen, Y., Danieli, S., et al. 2018a, ApJL, 856, L30 van Dokkum, P., Danieli, S., Cohen, Y., et al. 2018b, Natur, 555, 629 van Dokkum, P. G., Abraham, R., Merritt, A., et al. 2015, ApJL, 798, L45 Venhola, A., Peletier, R., Laurikainen, E., et al. 2017, A\&A, 608, A142 Yagi, M., Koda, J., Komiyama, Y., \& Yamanoi, H. 2016, ApJS, 225, 11 Zaritsky, D. 2017, MNRAS, 464, L110

Zaritsky, D., Crnojević, D., \& Sand, D. J. 2016, ApJL, 826, L9 